\title{
Fault Tolerant Control Strategy Using Two-Layer Multiple Adaptive Models for Plant Fault
}

\author{
Menglin HE \\ Electrical Engineering College \\ Guizhou University \\ 550025 Guiyang, China \\ LAAS-CNRS, Université de Toulouse \\ CNRS, INSA, UPS \\ 31400 Toulouse, France
}

\author{
Zetao LI \\ Electrical Engineering College \\ Guizhou University \\ 550025 Guiyang, China \\ gzgylzt@163.com
}

\author{
Boutaib DAHHOU \\ LAAS-CNRS, Université de Toulouse \\ CNRS, INSA, UPS \\ 31400 Toulouse, France
}

\begin{abstract}
Fault tolerant control (FTC) is always a popular research direction in the domain of automatic control. Inspired by the concept of adaptive model and corresponding approaches in [1], this paper proposed an FTC design strategy for plant fault by introducing these adaptive models into the two-layer multiple model structure. The two-layer multiple model structure describes a hyper-system which considers the nominal and faulty situations of a complex system. A group of local models are selected to present the system in its full range of operation and this is the first layer multiple model. At the second layer, we create a group of model bank to describe the system in nominal and each faulty situations. By checking the validity of the second layer model banks, information of corresponding local models are used to initialize the adaptive models to have a precise approaching to the real system. Besides, model predictive control (MPC) is designed for the reference model of the adaptive process to generate proper reference input for achieving control goals while dealing the FTC problem. Simulations are given to show the validity of the proposed method.
\end{abstract}

Index Terms-multiple model, adaptive control, fault tolerant control

\section{INTRODUCTION}

As we all know, automatic facilities are widely used and are also getting advanced and complicated. Security and stability performances are always strong demands for these systems to deteriorate the risks and losses caused by unexpected faults, especially in the domains of industry and traffic. For this reason, researches on Fault Detection and Diagnosis (FDD) [2] and FTC systems have attracted great attentions. FTC, that has the ability to guarantee the control performance in acceptable range in faulty situations [3] [4], is one of the most popular research direction in the domain of automatic control. FTC techniques are generally classified into active and passive ones [5]. Passive FTC strategies behave more like robust control, which are pre-designed and run without the module of neither real-time FDD nor control reconfiguration [6]. Active FTC, on the other hand, would automatically

This work was supported by China Scholarship Council (CSC), the National Natural Science Foundation of China (61963009), Science and Technology Planning Project of Guizhou Province ([2019]2154) and ([2016]2302), and Special fund project of provincial governor for outstanding science and technology education talents in Guizhou Province ([2010]4). adjust the control law using the fault information which is presented by a Fault Detection and Isolation (FDI) module and try to achieve the control goals with minimum performance degradation [7]. The active FTC approach is more flexible to apply in a big range of occasions while the passive approach is easy to design and implement since it doesn't need a FDD unit nor a reconfiguration mechanism[11].

Multiple model approach, for its simplicity in dealing with complex systems, has drawn increasing attention in the applications of engineering. It was originally proposed in [8] and was systematically described in [9]. Its drawbacks in bringing calculation burdens are gradually solved by the development of computing devices and parallel calculation techniques, which intensively boosts the growth of this method. It is now not only used for controller design [10], but is also applied in the domain of system reliability like fault diagnosis and fault tolerant control [11]. This approach deals with fault diagnosis problems in a way to avoid the complicated process of observer design. However, complexities still exist in its structure creation, parameter determination as well as integrated controller design and tuning for each sub-model, especially when the considered system is complex and highly nonlinear.

Adaptive control theory [12] [13], on the other hand, is very classic and has been studied since the 1960s form dealing with the control of linear time-invariant (LTI) systems with unknown parameters [14]. Classical adaptive control assures both stability and robustness when parametric errors of the considered system are small. When they are large, oscillations would appear in the transient response of adaptive systems. To overcome this, numerous and tremendous of efforts have been made. Among these efforts, combination with other theories is one of the popular direction. The concept and theoretical works propose in [1] are interesting combinations of multiple model approach and adaptive control. It also showed great improvements in solving the control problem of the system with unknown parameters.

This paper proposed a strategy for FTC design by introducing the multiple adaptive models concept into the twolayer multiple model structure which was presented before. 
During this process, multiple model approach is applied in two dimensions to form a two-layer multiple model structure for precise system representation in both nominal and the considered faulty situations. These two-layer multiple banks will then be used as a prior knowledge for the FTC implementation. By online checking the outputs of the first layer model banks and the real plant, faulty regime could be located. Decision variables would give the indication of current working point. In this way a group of local simple models could be confirmed and their parameters could be used to initiate the multiple adaptive models which will finally identify the faulty plant. Model Predictive Control (MPC) is selected to design a controller for the reference model to achieve the overall control goal by chasing the reference inputs.

This paper is organized as follows. Section II explains the two-layer model structure. Section III introduces the idea of adaptive models and presents the overall system structure with a controller for the reference model. Section IV states the FTC strategies and gives out the simulation results with discussions. A conclusion of this paper is then made in Section V.

\section{Two-Layer Multiple Model Structure}

\section{A. The First Layer Multiple Model}

Multiple model approaches have appeared more or less independently in several branches of science and engineering for a long time. In control domain, the divide-and-conquer strategy is implemented by multiple model approaches to deal with system complexities [9]. For a complex nonlinear system (1), which is the most common case in reality, one can always decompose its full range of operation into operating regimes.

$$
\left\{\begin{array}{l}
\dot{x}(t)=f(x(t), u(t)) \\
y(t)=C x(t)
\end{array}\right.
$$

where $x \in \mathbb{R}^{n}$ is the state, $u: \in \mathbb{R}^{m}$ is the system input. $f(\cdot)$ is a nonlinear function, $C \in \mathbb{R}^{p \times n}$ is the output matrix and $y \in \mathbb{R}^{p}$ is the output.

Within each regime, simple local model or controller structure could be selected. A method is applied to combine the local models or controllers into a global one and this combination of method and local models is defined as a model bank, e.g. (2).

$$
\left\{\begin{aligned}
\dot{x}_{i}(t) & =A_{i} x_{i}(t)+B_{i} u(t) \\
y_{i}(t) & =C_{i} x_{i}(t) \\
y(t) & =\sum_{i=1}^{L} \mu_{i}(t) y_{i}(t)
\end{aligned}\right.
$$

where $A_{i} \in \mathbb{R}^{n \times n}, B_{i} \in \mathbb{R}^{n \times m}$ and $y_{i} \in \mathbb{R}^{p}$ are the system , input matrices and output of $i^{t h}$ local model respectively. $\mu$ is the weight and $y$ is the overall output of the model bank.

In this way, the problem to a complex system is conquered indirectly. We call these local models the first layer multiple model in this paper. There are a lot of methods to obtain multiple model expression for a complex system, local linearization, for example, is a traditional way. On the other hand, system identification could also help to achieve a model bank with high accuracy [15].

\section{B. The Second Layer Multiple Model Bank}

If we do a simple extension and think about the faulty situations, we could expand the concept of multiple model approach to the second dimension. A fault in a dynamical system is defined as a deviation of the system structure (e.g. actuator or sensor fault) or the system parameters (e.g. plant fault) from the nominal situation [1]. From this point of view, parameter could be considered as a variable to a hyper-system (or a time-variant system) which contains plant fault (3).

$$
\left\{\begin{array}{l}
\dot{x}(t)=f(x(t), \theta(t), u(t)) \\
y(t)=C x(t)
\end{array}\right.
$$

where $\theta$ is the system parameter which might be changed by a fault.

When a system in nominal situation is described by the first layer multiple models, we define the combination of local models as the nominal model bank. In the same way, the system in faulty situation could also own its model bank and it is called the faulty model bank. Thus, under the axis of fault, a series of model banks could be constructed by dividing the fault regimes. Here we define it as the second layer multiple model bank. In this paper, we consider the case whose twolayer multiple model banks could be created off-line and set as a priory for the following sections. As an example, to decomposed a system like (3), a two-layer multiple model banks may has the form of (4) and the structure is illustrated in Fig. 1.

$$
\left\{\begin{array}{l}
\dot{x}_{i j(t)}=A_{i j} x_{i j(t)}+B_{i j} u(t) \\
y_{i j(t)}=C_{i j} x_{i j(t)} \\
y_{j}(t)=\sum_{i=1}^{L} \mu_{i j(t)} y_{i j(t)}
\end{array}\right.
$$

where $j$ indicates the considered faulty situation.

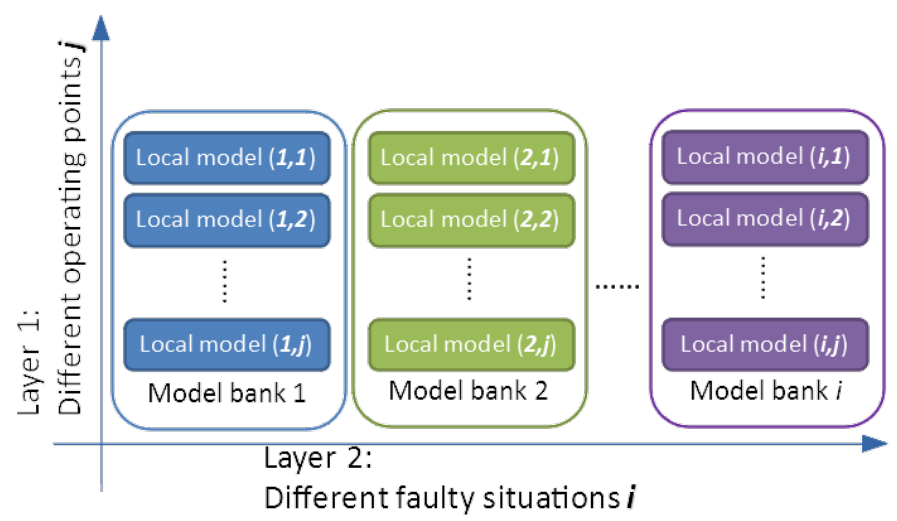

Fig. 1. Two-layer multiple model structure. 


\section{Multiple Adaptive Models}

\section{A. Adaptive Control Problem and Identification Model}

This part of theoretical work was originally presented in [1]. The concept and main idea is introduced here and combined with the two-layer multiple model structure for dealing the FTC problem. Generally, sub-models obtained in the previous section are simple and easy to use. We assume these local models are linear time-invariant ones and their state variables are accessible. The real system at every moment could be described by the state equation that has the same structure as the local model in the model banks. For simplicity, we assume it has a special form:

$$
\dot{x}_{p}(t)=A_{p} x_{p}(t)+b u(t)
$$

where $A_{p} \in \mathbb{R}^{n \times n}$ and $b \in \mathbb{R}^{n}$ are in companion form. The last row of $A_{p}$ are $\left[a_{p(1)}, a_{p(2)}, \ldots, a_{p(n)}\right]=\theta_{p}^{T}$ and are assumed to be unknown. $b=[0, \ldots, 0,1]^{T}$.

A reference model will have the following description:

$$
\dot{x}_{m}(t)=A_{m} x_{m}(t)+b r(t)
$$

where $r(\cdot): \mathbb{R}^{+} \rightarrow \mathbb{R}$ is a bounded piece-wise continuous reference signal to assure the adaptive process satisfy the overall control goal. It will be discussed later. $A_{m}$ is also in companion form and has the last row $\theta_{m}^{T}$.

The reference model is generally stable and its parameters are known. It stands for the real plant in the situation which we expected. Thus, the objective of adaptive control is to calculate the input $u(\cdot)$ to the real plant such that $\lim _{t \rightarrow \infty}\left[x_{p}(t)-\right.$ $\left.x_{m}(t)\right]=0$. To achieve this, the following identification model is set up:

$$
\dot{x}_{I}(t)=A_{m} x_{I}(t)+\left[A_{I}(t)-A_{m}\right] x_{p}(t)+b u(t)
$$

where $A_{I}(t)$ is a matrix in companion form and its last row $\theta_{I}^{T}(t)=\left[a_{I(1)}, a_{I(2)}, \ldots, a_{I(n)}\right]$ contains the identified parameters and is adaptable.

Defining $\tilde{\theta}_{I}(t) \triangleq \theta_{I}(t)-\theta_{p}$ and $e_{I}(t) \triangleq x_{I}(t)-x_{p}(t)$, the well known adaptive law is given:

$$
\dot{\theta}_{I}(t)=-e_{I}^{T}(t) P b x_{p}(t)
$$

where $P$ is the positive definite matrix solution of the Lyapunov equation $A_{m}^{T} P+P A_{m}=-Q, Q=Q^{T}>0$.

Equation (8) makes a Lyapunov function candidate

$$
V\left(e_{I}, \tilde{\theta}_{I}\right)=e_{I}^{T} P e_{I}+\tilde{\theta}_{I}^{T} \tilde{\theta}_{I}
$$

having a result in $\dot{V}\left(e_{I}, \tilde{\theta}_{I}\right)=-e_{I}^{T} Q e_{I}<0$, which assures the boundedness of $\tilde{\theta}_{I}(t)$ and $e_{I}(t)$. Therefore, the following feedback control is used to assure the stability of the plant:

$$
u(t)=-k^{T}(t) x_{p}(t)+r(t)
$$

where $k(t)=\theta_{I}(t)-\theta_{m}$.

\section{B. Controller Design for Reference Model}

The previous sub-section discussed how adaptive law is used to create an adaptive model for identifying the unknown system. The calculated control signal is applied on the system and force it approaching the states and outputs of the reference model. As we described before, a known reference signal is needed here and it is set as the input of both the reference model and the adaptive controller. However, this reference signal is not the initial reference input which is the control goal of the whole system. In order to identify the real plant, approach the reference model and achieve the control goal, a controller should be designed for the reference model. As is shown in Fig. 2, MPC is introduced here to accomplish this task.



Fig. 2. Introduction of MPC for reference model

Under this architecture, the adaptive law focuses on identifying the real system and chasing the reference model while the MPC controller cares about the initial reference input. Since the reference model (6) is known, its MPC controller is very simple to design [16]:

$$
r(t)=\int_{0}^{t} \dot{u}_{m p c}(\tau) d \tau
$$

where $\dot{u}_{m p c}$ is given by:

$$
\dot{u}_{m p c}(t)=\left[\begin{array}{ll}
K_{x} & K_{y}
\end{array}\right]\left[\begin{array}{c}
\dot{x}_{m}(t) \\
y_{m}(t)-r_{i n}(t)
\end{array}\right]
$$

where $\left[\begin{array}{ll}K_{x} & K_{y}\end{array}\right]=K_{m p c}$ is the MPC gain. $x_{m}(t)$ and $y_{m}(t)$ are the state and output of the reference model. $r_{i n}(t)$ is the initial reference input.

\section{Extension to Multiple Adaptive Models}

When simultaneously running the two-layer multiple banks, the real system will always be surrounded by several local models in the two-dimension netted space. Information of these models are useful because they are close to the real situation and can help launching a fast search. In adaptive control domain, it is allowed to use any number of models to identify the system but only one control signal can be applied. Thus, we do an extension of (7) and introduce all the $N$ surrounding local models into the identification process.

$$
\left\{\begin{array}{l}
\dot{x}_{l}(t)=A_{m} x_{l}(t)+\left[A_{l}(t)-A_{m}\right] x_{p}(t)+b u(t) \\
x_{l}\left(t_{0}\right)=x_{p}\left(t_{0}\right)
\end{array}\right.
$$


where $l \in[1, N]$ indicates $l^{t h}$ identification model.

The strategy to organize them is simple: the one who owns the minimum estimation error wins and the control calculation will be based on its information. So the new control signal would still be given by (10) but the calculation of $k(t)$ will be different and is given in (14).

$$
k(t)=\theta_{z}(t)-\theta_{m}
$$

where $z$ is current index of minimum $e_{l}(t)$.

Parameter renew of each identification model is given in (15).

$$
\dot{\theta}_{l}(t)=-e_{l}^{T}(t) P b x_{p}(t)
$$

\section{FTC STRATEGY AND SimULATION EXAMPLES}

\section{A. FTC Strategy}

So far, the three elements, namely the two-layer multiple model banks, $N$ adaptive models and controller of reference model, have been prepared. The following Two-layer Multiple Adaptive Model Based FTC strategy could be given:

\section{- Start:}

- Require: Mathematical model of the targeting system (3)

- Require: IO data generated by running system (3) in nominal and faulty situations

- Require: Two-layer multiple model banks (4)

- Require: Parameters of the reference model (6)

- Require: MPC controller (11), (12) design for the reference model

\section{- 1. Initial setting}

- 2. Repeat

- 3. Run reference model to generate current $y_{m}(t)$

- 4. Calculate $r(t)$ according to (11) and (12)

- 5. Run multiple model bank to generate current $y_{j}(t)$

- 6. Compare $y_{p}(t)$ with all $y_{j}(t)$ and give out index $j(t)$ and $j(t)+1$ of two neighboring model banks who contain current plant

- 7. Check the decision variables (which could be set as input, states or output according to the multiple model banks) to give out index $i(t)$ and $i(t)+1$ of two neighboring working points who contain current plant

- 8. if $j(t) \neq j(t-1)$ or $i(t) \neq i(t-1)$

- 9. then use $A_{i, j}, A_{i+1, j}, A_{i+1, j+1}, A_{i, j+1}$ in (4) and $A_{m}$ in (6) to initiate 4 identification models (7)

- 10. Calculate $e_{l}(t)=x_{l}(t)-x_{p}(t)$ and give out the index $z$ of minimum $e_{l}(t)$

- 11. Calculate $k(t)$ using (14)

- 12. Calculate control signal $u(t)$ using (10) and apply it to run the plant and identification models the next period

- 13. Renew parameters of each identification model using (15)

- 14. if stop condition is satisfied

- 15. then go ahead to stop else go to Repeat

- 16. else go to Repeat

- End

\section{B. Simulations Conditions and Parameters}

For the strategy proposed above, simple simulations are shown. In these simulations, we assume that the two-layer multiple model bank has be created and parameters of the local models are known. The reference model is chosen to have $\theta_{m}=[-1.5,-2.5]^{T}$ all the time (16).

$$
\left\{\begin{array}{l}
\dot{x}_{m}(t)=\left[\begin{array}{cc}
0 & 1 \\
-1.5 & -2.5
\end{array}\right] x_{m}(t)+\left[\begin{array}{l}
0 \\
1
\end{array}\right] r(t) \\
y_{m}(t)=\left[\begin{array}{ll}
1 & 0
\end{array}\right] x_{m}(t)
\end{array}\right.
$$

The real plant in nominal situation is initially presented by a stable second order system (17) whose parameter $\theta_{p}=$ $[-2.1,-1.5]^{T}$ and there are always 4 surrounding local models in the parameter space (18). Output of the system is set to be the first state, namely, matrix $C=[1,0]$.

$$
\begin{aligned}
& \left\{\begin{array}{l}
\dot{x}_{p}(t)=\left[\begin{array}{cc}
0 & 1 \\
-2.1 & -1.5
\end{array}\right] x_{p}(t)+\left[\begin{array}{l}
0 \\
1
\end{array}\right] u(t) \\
y_{p}(t)=\left[\begin{array}{ll}
1 & 0
\end{array}\right] x_{p}(t)
\end{array}\right. \\
& \left\{\begin{aligned}
& \dot{x}_{l}(t)= {\left[\begin{array}{cc}
0 & 1 \\
-1.5 & -2.5
\end{array}\right] x_{l}(t)+\left[\left[\begin{array}{cc}
0 & 1 \\
\theta_{l, 1}(t) & \theta_{l, 2}(t)
\end{array}\right]-\right.} \\
& {\left[\begin{array}{cc}
0 & 1 \\
-1.5 & -2.5
\end{array}\right] x_{p}(t)+\left[\begin{array}{l}
0 \\
1
\end{array}\right] u(t) } \\
& y_{l}(t)=\left[\begin{array}{ll}
1 & 0
\end{array}\right] x_{l}(t)
\end{aligned}\right.
\end{aligned}
$$

where $l \in[1,4]$ and $\theta_{l}(t 0)$ is initiated from $\theta_{11}=[-3,3]^{T}$, $\theta_{12}=[3,3]^{T}, \theta_{21}=[-3,-3]^{T}$ and $\theta_{22}=[3,-3]^{T}$ respectively.

When running the FTC simulations, a plant fault is introduced and the parameter of the corresponding system will change to $\theta_{p f}=[6.9,5.1]^{T}$, which are big deviations in system parameters as is shown in (19).

$$
\left\{\begin{array}{l}
\dot{x}_{p, f}(t)=\left[\begin{array}{cc}
0 & 1 \\
6.9 & 5.1
\end{array}\right] x_{p, f}(t)+\left[\begin{array}{l}
0 \\
1
\end{array}\right] u(t) \\
y_{p, f}(t)=\left[\begin{array}{ll}
1 & 0
\end{array}\right] x_{p, f}(t)
\end{array}\right.
$$

The surrounding models in this situation have the same structure as (18) but their parameters are initiated from $\theta_{11}=$ $[3,3]^{T}, \theta_{12}=[3,9]^{T}, \theta_{21}=[9,9]^{T}$ and $\theta_{22}=[9,3]^{T}$. At each moment, the 4 models would generate a virtual square surrounding the real plant.

\section{Simulations without Fault}

To investigate the efficiency of single identification model and multiple adaptive models, fault is temporarily not introduced. A square-wave with a period time of $37.5 \mathrm{~s}$ is set as the initial reference input and MPC is constructed for the reference model. First, a single model with $\theta_{11}=[-3,3]^{T}$ is chosen to initiate the identification model and launch the adaptive law. Simulation results are shown in the following Fig. 3.

From Fig. 3, it is clear that the MPC controller works well. Output of the reference model could follow the changing initial reference input even if it is not given in square-wave. Overshoots in the output of reference model $y_{m}$ could be adjusted by tuning parameters of the MPC controller. This 
(a) Reference inputs and outputs of systems



(b) Outputs of controllers

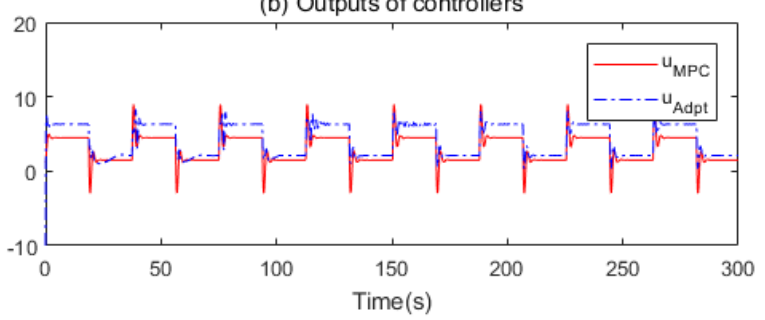

Fig. 3. Single adptive model case without fault.

controller also gives out the intermediate reference signal $u_{m p c}$ which is renamed as $r(t)$ and applied both to the reference model and the adaptive law as is shown in Fig. 2.

We can also notice that output of the real system $y_{p}$ is approaching that of the reference model gradually. It is relatively large at first, especially in the overshoot area. And it converged after about 150 seconds. This indicates that the adaptive model works also, even though the identification speed is not ideal.

After that, full list of the 4 surrounding local models in nominal situation is applied. Fig. 4 presents the comparison of output errors between plant and reference model in single adaptive model case and multiple adaptive models case.

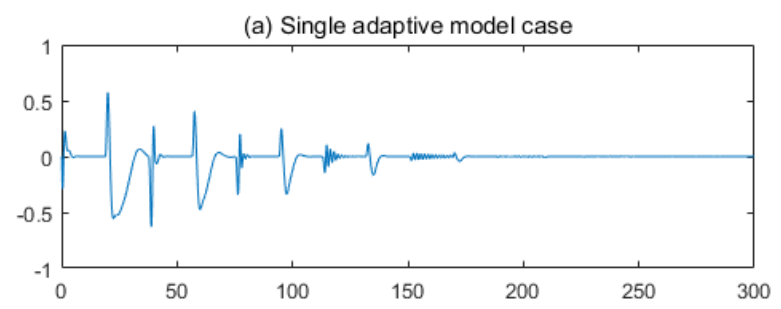

(b) Multiple adaptive models case

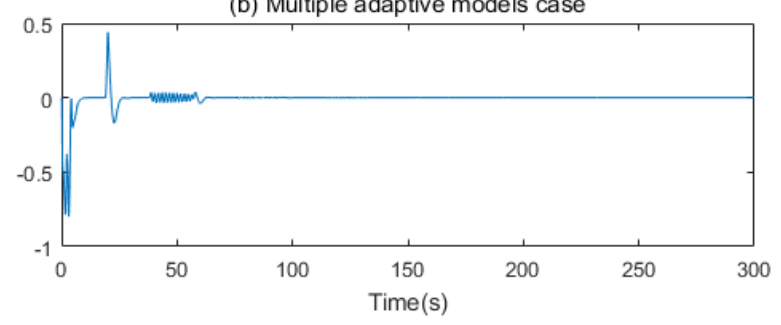

Fig. 4. Comparison of output errors between plant and reference model in single adaptive model case and multiple adaptive models case.

Apparently, by fully using information of the surrounding models to initiate the adaptive models, identification speed is accelerated in a large scale. It takes only 50 seconds to approach the unknown real system.

\section{Fault Tolerant Control Simulations}

It is sure now that performance of multiple adaptive models is superior than the single case. Thus we simulate a faulttolerant case under the proposed strategy. A plant fault is introduced at time $T=124 \mathrm{~s}$. As described in the beginning of this section, this fault leads to a change in system parameters. By checking the output of the plant and model banks, we will have an idea about the fault's extent and give out the two neighboring model banks. And the value of decision variable would used to be the second coordinate and locate the regime of working point. Therefore, the four surrounding local models could be given. New adaptive models are constructed accordingly.

(a) Reference inputs and outputs of systems

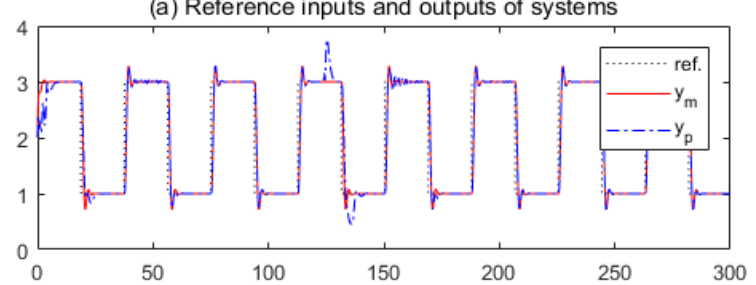

(b) Outputs of controllers

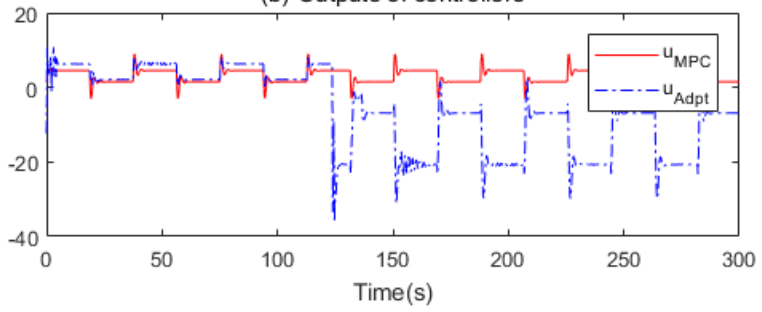

Fig. 5. FTC Simulation(plant fault is introduced at time $T=124 s$ ).

Fig. 5 shows the simulation results of the proposed strategy. In normal situation, the adaptive law will quickly adjust the unknown system to approach the reference model. MPC of the reference model gives proper reference signal $r(t)$ to assure the final outputs of the system follow the given initial reference inputs, i.e. the square-wave signal. After the introduction of the plant fault, the stable states are broken and big oscillations are very obvious in the figure. However, the proposed strategy quickly adjusted and calmed down the influence of the fault. It takes about 50 seconds to achieve this. As is shown in Fig. 5 (b), to follow the same reference inputs, control signal which is given by the adaptive law is quite different from that of the MPC controller. That is reasonable because the plant parameters changed a lot and are no longer as close to the unchanged reference model as before.

Fig. 6 presents the error curves of the FTC simulation. Subfigure (a) is the errors between the outputs of the reference model and the given initial reference inputs. It shows there's still improvement space for optimizing the MPC parameters. The big overshoots is caused by the time delay for 
(a) Errors between reference model and reference inputs

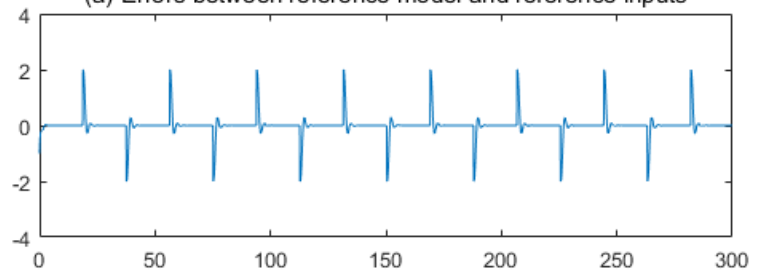

(b) Output errors between plant and reference model

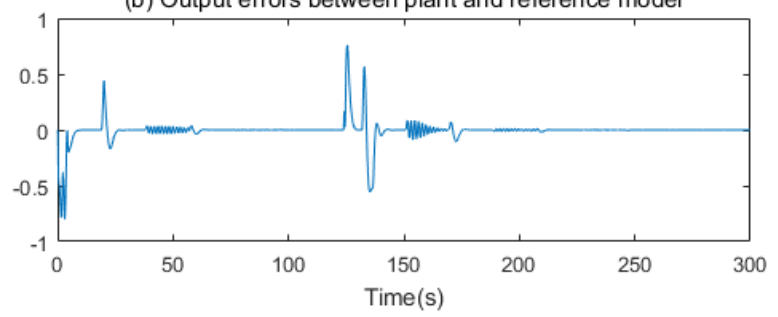

Fig. 6. Error curves of the FTC Simulation.

detecting the change of reference inputs. Since this signal is set manually, we could adjust the rule and give it to the controller one step ahead to avoid. Sub-figure (b) shows the output errors between the system and the reference model. It is clearly telling us when the fault is introduced and when the identification process is converged, which supported the discussion given before. This simulation showed the validity of the proposed strategy.

\section{CONCLusions}

This paper presented a FTC design strategy for plant fault. It firstly described the concept of the two-layer multiple model structure which is used to simplify the complex system and makes it accessible to each region of the hyper-system concerning fault. Secondly it introduced the idea of multiple adaptive models which have the ability to assure convex hull property. Combinations of the two concepts are described and a MPC controller is added to generating proper intermediate reference signal while achieving the overall control goal. Simulations showed that multiple adaptive models obviously have a better performance than the single one, that it took far less time for convergence. This idea is perfect for the two-layer multiple model structure. The FTC simulation showed the validity of the proposed strategy that the system can achieve control goal and follow the reference input after a short period of adjusting when a plant fault occurs.

\section{REFERENCES}

[1] Z. Han, K. S. Narendra. New concepts in adaptive control using multiple models. IEEE Transactions on Automatic Control, 2011, 57(1): 78-89.

[2] R. Isermann. Fault-diagnosis applications: model-based condition monitoring: actuators, drives, machinery, plants, sensors, and fault-tolerant systems. Springer Science Business Media, 2011.

[3] M. Blanke, M.Kinnaert, J. Lunze, M. Staroswiecki, and J. Schröder. Diagnosis and fault-tolerant control. Berlin: Springer, 2006

[4] H. Yang, B. Jiang, M. Staroswiecki. Supervisory fault tolerant control for a class of uncertain nonlinear systems. Automatica, 2009, 45(10): 2319-2324.
[5] Z. Li, B. Dahhou, Q. Li, M. Zhang. Design of passive fault tolerant control of a process system. The 27th Chinese Control and Decision Conference (2015 CCDC). IEEE, 2015: 2776-2781.

[6] X. Yu, Y. Zhang. Design of passive fault-tolerant flight controller against actuator failures. Chinese Journal of Aeronautics, 2015, 28(1): 180-190.

[7] D. Rotondo, F. Nejjari, V. Puig. Passive and active FTC comparison for polytopic LPV systems. 2013 European Control Conference (ECC) IEEE, 2013: 2951-2956.

[8] D. Magill. Optimal adaptive estimation of sampled stochastic processes. IEEE Transactions on Automatic Control, 1965, 10(4): 434-439.

[9] R. M. Smith, T. A. Johansen. Multiple model approaches to modelling and control. Britsh Library Cataloguing in Publication, Taylor Francis, 1997.

[10] T. D. Murphey. On multiple model control for multiple contact systems. Automatica, 2008, 44(2): 451-458.

[11] A. Mirzaee, K. Salahshoor. Fault diagnosis and accommodation of nonlinear systems based on multiple-model adaptive unscented Kalman filter and switched MPC and H-infinity loop-shaping controller. Journal of Process Control, 2012, 22(3): 626-634.

[12] K. S. Narendra, A. M. Annaswamy. Stable adaptive systems. Courier Corporation, 2012.

[13] G.C. Goodwin, K. S. Sin. Adaptive filtering prediction and control. Courier Corporation, 2014.

[14] Z. Han, K. S. Narendra. Second level adaptation using multiple models. Proceedings of the 2011 American Control Conference. IEEE, 2011: 2350-2355.

[15] R. Orjuela, B. Marx, J. Ragot, D. Maquin. Nonlinear system identification using heterogeneous multiple models. International Journal of Applied Mathematics and Computer Science, 2013, 23(1): 103-115.

[16] L. Wang. Model predictive control system design and implementation using MATLAB. Springer Science Business Media, 2009. 